\title{
High Ferritin Before Transplant Increases Cytopenia Post-transplant in HLA Mismatched Hematopoietic Stem Cell Transplantation in $\beta$-Thalassemia Major
}

\author{
Liao Jianyun, Issa Moussa Mardo, Wen Jianyun, Zhou Xiaohui, Peng Zhiyong, Liu Huaying, \\ Chen Libai, He Yuelin, Pei Fuyu, Wu Xuedong, Feng Xiaoqin, Bai Jing, Su Qingxia, Li Chunfu ${ }^{*}$ \\ Department of Pediatrics, Nanfang Hospital, Southern Medical University, Guangzhou, China
}

Email address:

chunfugzcn@126.com(Li Chunfu)

${ }^{*}$ Corresponding author

To cite this article:

Liao Jianyun, Issa Moussa Mardo, Wen Jianyun, Zhou Xiaohui, Peng Zhiyong, Liu Huaying, Chen Libai, He Yuelin, Pei Fuyu, Wu Xuedong, Feng Xiaoqin, Bai Jing, Su Qingxia, Li Chunfu. High Ferritin Before Transplant Increases Cytopenia Post-transplant in HLA Mismatched Hematopoietic Stem Cell Transplantation in $\beta$-Thalassemia Major. American Journal of Pediatrics. Vol. X, No. X, 2018, pp. XX-XX. doi: 10.11648/j.ajp.20180402.13

Received: April 13, 2018; Accepted: May 24, 2018; Published: June 14, 2018

\begin{abstract}
The objective is to find out the causes of Cytopenia post-engraftment (CPE) in hematopoietic stem cell transplantation (HSCT) for patients with $\beta$-thalassemia major ( $\beta$-TM). For this, 61 consecutive $\beta$-TM patients underwent $7 / 8$ HLA matched HSCT from January 1, 2009 to June 30, 2015 were retrospectively analyzed. Thirty-eight patients suffered from $\mathrm{CPE}$ and the remainder $(\mathrm{n}=23)$ associated without CPE. The effect of pre-transplant ferritin (PTF) and recipient and donor age on CPE were analyzed in the two groups. The CPE was defined as white blood cell counts less than 3.0 $\times 109 / \mathrm{L}$ for four weeks or longer without infection of cytomegalovirus, human parvovirus B19 virus and Epstein-Barr virus. As result, in univariate analysis, PTF level was a high-risk factor for CPE and significant higher in CPE group than no-CPE group (3927.9 \pm 1314.9 vs $2291.0 \pm 994.4 \mathrm{ng} / \mathrm{ml}, \mathrm{p}=0.000)$. The result was also proved by multi-factor binary regression analysis $(\mathrm{p}=0.001)$. The optimal value of PTF level by R language ( $R$ 2.15.2) is $2500 \mathrm{ng} / \mathrm{ml}$, which is cutoff value in the two groups. The current study showed high PTF level was a high-risk factor of CPE for $\beta$-TM patients underwent 7/8 HLA-matched HSCT. PTF should be reduced below $2500 \mathrm{ng} / \mathrm{L}$ if the $7 / 8$ matched HSCT must be done.
\end{abstract}

Keywords: Ferritin, Cytopenia, Thalassemia, HSCT

\section{Introduction}

$\beta$-thalassemia major $(\beta-\mathrm{TM})$ is an ineffective hematopoietic or hemolytic anemia that results in an imbalanced accumulation of alpha-globin chains due to reduced or absent beta-globin synthesis [1, 2]. Although routine blood transfusions and iron-chelating therapy can reduce long-term complications and increase life expectancy [3], as well as the gene therapy just started is likely to cure thalassemia [4], allogeneic hematopoietic stem cell transplantation (HSCT) remains an only proved cure for thalassemia [5]. In thalassemia HSCT, HLA matched sibling is the optimal donor choice, but only about one third of patients benefit from it. In the absence of matched sibling donor, mismatched (7/8) related and unrelated donor and cord blood transplants (alternative donor transplant, ADT) have been studied [6, 7]. However, more complication, such as infections, cytopenia post engraftment (CPE) and graft versus host disease (GVHD) have been observed in ADT. Of them CPE is one of the commonest complication after ADT. The possible causes include infection, GVHD, medication and pre-transplant factors (pre-transplant status, donor age, transplant type, and HLA matched degree) [8-11].

\section{Patients and Methods}

\subsection{Researched Object}

Data of 61 patients with $\beta$-TM underwent 7/8 HLA matched (at HLA-A, -B, -C or -DRB1 antigens or allele levels) related or 
unrelated donor HSCT from January 1, 2009 to June 30, 2015 were collected in the center. Parents of all patients included in this study signed informed consent in accordance with the Declaration of Helsinki prior to HSCT and discussed in detail with the doctor the basis for the risk / benefit ratio of transplantation and possible complications, including loss of fertility. Sixty-one patients were divided into CPE group $(\mathrm{n}=38)$ and CPE-free group $(\mathrm{n}=23)$ according to the presence or absence of CPE. Pre-transplant characteristics of two groups patients are shown in Table 1. All patients were classified as degree II status at HSCT time according to Nanfang Hospital's standard [12].

Table 1. Patient and Transplant characteristics.

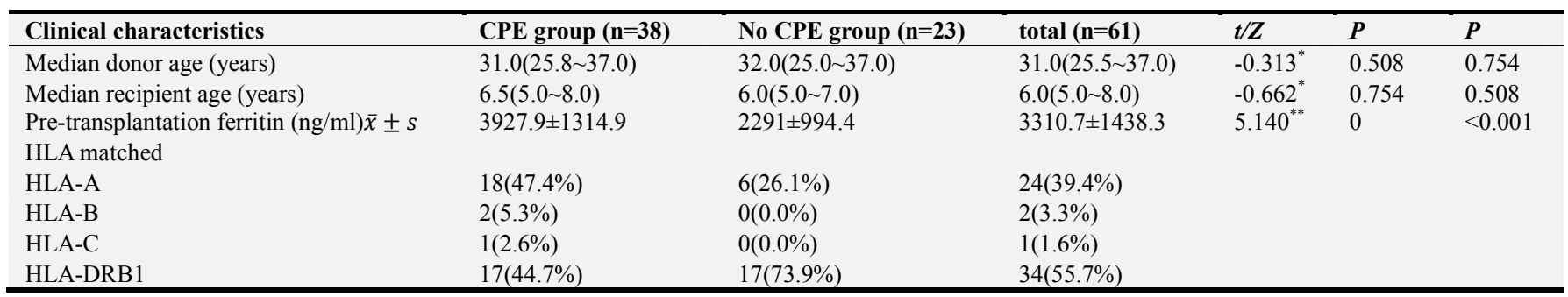

Note:\# CPE, cytopenias after days 28 transplantation; *Mann- Whitney U- test, ** Independent sample T- test.

\subsection{Conditioning Regimen and GVHD Prophylaxis}

All patients received the uniform NF-08-TM conditioning regimen [12], Including intravenous busulfan, cyclophosphamide, fludarabine, and thiotepa, and the dose was adjusted according to the status before transplant by NF-08-TM protocol. Prophylaxes of GVHD included cyclosporine A (Cs-A) starting with intravenous injection of $1.5 \mathrm{mg} /(\mathrm{kg} \cdot \mathrm{d})$ from days -10 to -2 and then increasing to 3 $\mathrm{mg}$ on days -1 to $25 /(\mathrm{kg} \cdot \mathrm{d})$, and subsequently administration orally at targeted concentration of $200 \pm 50 \mathrm{ng} / \mathrm{ml}$. The dose of Cs-A gradually decreased from the 60th day until it was terminated at the end of 1 year after transplantation. Mycophenolate Mofetil (MMF) was administered at a dose of $15 \mathrm{mg} / \mathrm{kg}$ bid on the first day after transplantation and stopped on the $30^{\text {th }}$ day if there was no sign of grade II or more acute GVHD. Thymoglobuline (France) was used at $2.5 \mathrm{mg} /$ $(\mathrm{kg} \cdot \mathrm{d})$ from day -3 to day -1 . In addition, short-term methotrexate was administered on days 1,3 and 6 at doses of 15,10 and $10 \mathrm{mg} / \mathrm{m} 2$, respectively. All patients received the same standard cell dose $\left(8 \times 10^{8} / \mathrm{kg}\right)$ of granulocyte colony stimulating factor (G-CSF) primed peripheral blood stem cells.

\subsection{Support Treatment}

All of the patients were given anti-cytomegalovirus (CMV) pre-transplant emptying treatment, the CMV status was monitored weekly by quantitative PCR for 6 months, and patients with CMV reactivation were treated with ganciclovir or foscarnet. G-CSF was not administered to any patients. The threshold for red blood cell and platelet transfusion were hemoglobin $8 \mathrm{~g} / \mathrm{L}$ and platelet $20 \times 10^{3} / \mathrm{mm}^{3}$, respectively. For patients with iron overload, no iron-chelating therapy should be used within one year of transplantation or prior to discontinuing immunosuppressive drugs.

\subsection{CPE Definition}

Inclusion criteria: (1) peripheral blood white blood cells $<3.0 \times 10^{9} / \mathrm{L}$ for 4 weeks or more; (2) neutrophil count $<0.5 \times 10^{9} / \mathrm{L}$ for 4 weeks or more; (3) $\mathrm{Hb}<80 \mathrm{~g} / \mathrm{L}$ for 4 weeks or more; (4) PLT $<20 \times 10^{9} / \mathrm{L}$ for 4 weeks or more; (5) Autoimmune hemolytic anemia (AIHA) with a clear clinically hemolysis and Coombs test positive .Exclusion criteria: (1) CPE with CMV quantitation greater than 500 copies / ml by PCR monitoring; (2) CPE during ganciclovir; (3) CPE caused by other viral infections such as parvovirus B19, Epstein-Barr virus and herpes virus infection. All CPE due to graft failure were not included in the study.

\subsection{Statistical Methods}

The characteristics of thalassemia patients were summarized using counts and percentages for this paper. When continuous variables are not normally distributed, the median and quartile measurements are used. When it is normal distribution, statistical measurements are made with the mean and standard deviation. A single-sample $\mathrm{T}$ test is used when continuous variables are normal, otherwise Mann Whitney $U$ test is used. Multivariate Regression Models Hazard ratios for assessing risk factors associated with CPE and $95 \%$ confidence intervals $(95 \%$ CI) Variables with significance levels less than 0.05 in univariate models are an optional part of the multivariate model. In order to determine the best fit set logarithm for any $\tau$ value in any model, log likelihood function values for the largest logarithmic part are recorded. The value of $\tau$ that produces the largest logarithm part likelihood function value is the optimal value of $\tau$. This procedure is completely illustrated in the PTF level of the $R$ language ( $R$ 2.15.2). The study used the SPSS 13.0 software package (SPSS) for analysis. P value $<0.05$ was considered to be statistically significant.

\section{Result}

All patients were successfully engraftment. Among them, CPE occurred in 38 cases. The cumulative incidence of CPE in one year was $62.4 \%(95 \% \mathrm{CI}, 33.7 \%-69.2 \%)$. The median time to CPE was 153 days after transplantation (range, 44th to 
196th days) and the duration of CPE was 92 days (range, 29 to 308 days). Of the 38 patients with CPE, 33 showed only varying degrees of leukopenia (leukopenia less than $3 \times 10^{9} /$ $\mathrm{L}, 2 \times 10^{9} / \mathrm{L}$, and $1 \times 10^{9} / \mathrm{L}$ in 17,11 and 5 cases, respectively), Two patients had thrombocytopenia, Three patients had leukopenia with thrombocytopenia. Thirty patients among 34 patients with leukopenia had full recovery of leukocyte count by adjusting dose of Cs-A and / or using MMF and tacrolimus. Another two cases with leukopenia received Thymoglobuline (France, $5 \mathrm{mg} / \mathrm{kg}$ ). Of them one resulted in normal leukocyte count and the other one had still abnormal white cell counts around $2 \times 10^{9} / \mathrm{L}$. In addition, the remaining two cases of leukopenia persisted for 3 months and did not return to normal levels by the end of the study.

In univariate analysis, the study identified PTF as an important risk factor of CPE. T test results from an independent sample showed the normal distribution of PTF. PTF levels in the CPE group were significantly higher than those in the CPE-free group (3927.9-1314.9 ng / ml vs 2291.0 -994.4 ng / $\mathrm{ml}(\mathrm{P}<0.001$; Table 1, Figure 1). Age-specific data for the donors and recipient following the abnormal distribution were evaluated using the Mann Whitney U test. Patients in the CPE group and in the CPE-free group had no significant difference in donor age (31.0 vs. 32.0, $\mathrm{P}=0.508$; Table 1, Figure 3 ) and recipient age (6.5 vs. $6.0, \mathrm{P}=0.754$, Table 1 , Figure 2$)$; PTF was also proved to be a risk factor by multivariate logistic regression model $(\mathrm{P}=0.001$; Table 2$)$. According to Figure 4, the optimal PTF level in R language ( $\mathrm{R}$ 2.15.2; Figure 4) is $2594 \mathrm{ng} / \mathrm{ml}$ and has a variable range of 2500-3500 $\mathrm{ng} / \mathrm{ml}$.

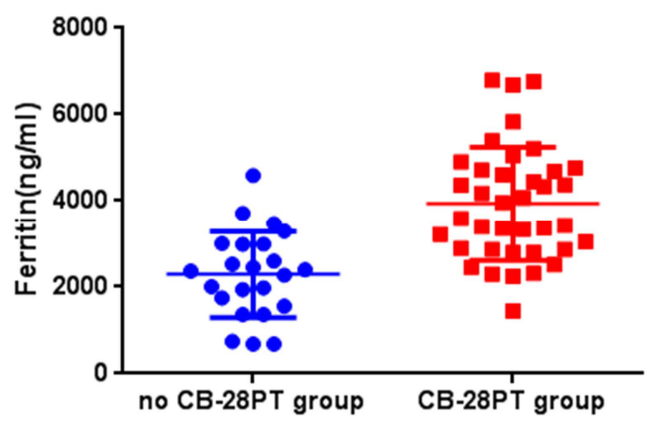

Figure 1. pre-transplant ferritin in CPE groupe were significantly higher than those in the no CPE group.

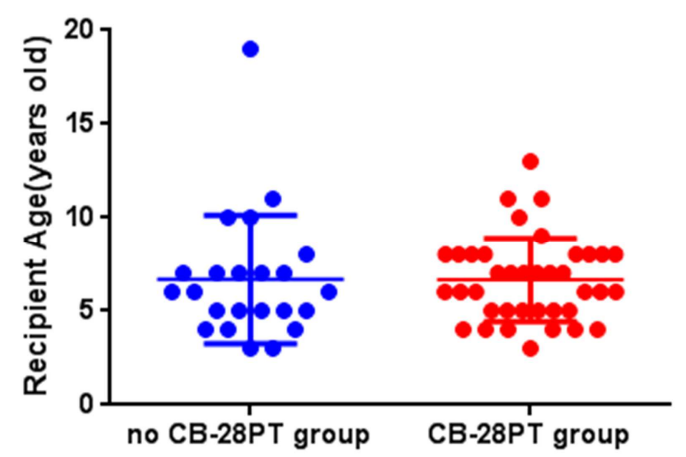

Figure 2. Patients in the CPE group and in the no CPE had no significant difference in recipient age.

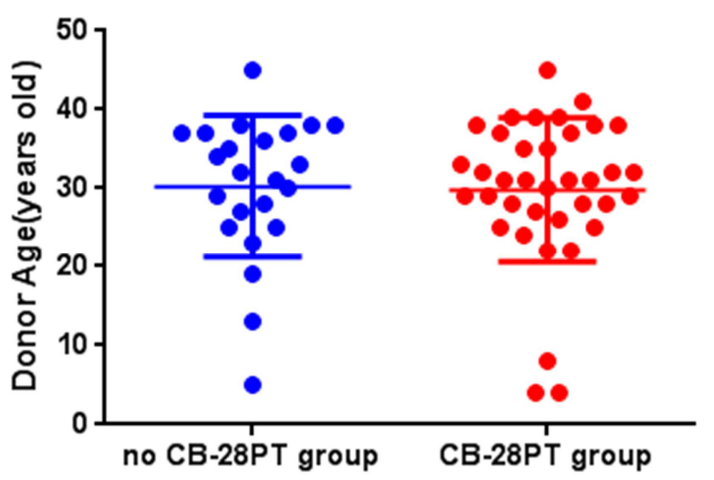

Figure 3. CPE group and in the no CPE group had no significant difference in donor age.

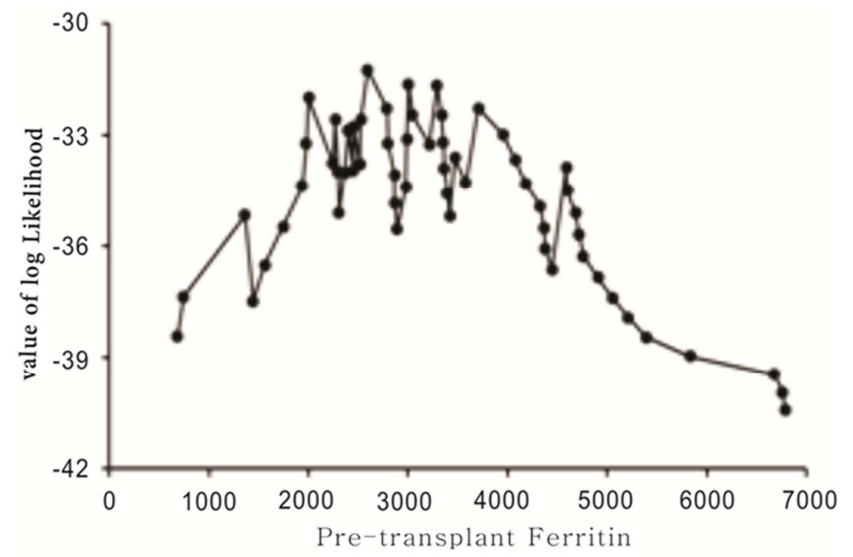

Figure 4. The optimal PTF level in R language is $2594 \mathrm{ng} / \mathrm{ml}$ and has a variable range of $2500-3500 / \mathrm{ml}$.

\section{Discussion}

The first reports of adverse effects of iron overload on transplant outcomes were found in a large study on patients who underwent multiple transfused thalassemia prior to transplantation [13]. Iron overload is currently recognized as a poor prognostic factor in HSCT. A variety of early transplant complications including infection, abnormal liver function, and sinusoidal obstructive syndrome have been attributed to iron overload. And it is also consensus that HSCT should be performed with the closest to normal value of ferritin in the body. However, the safety threshold of iron overload for different type transplant is unclear. This is also the purpose of the current study.

The occurrence of CPE seems to be associated with poor immune recovery after HSCT, namely, an imbalance between self-regulatory and autoreactive lymphocytes [14]. The clinical manifestations of CPE are usually similar to autoimmune diseases [15]. However, the exact pathophysiology of CPE is still not fully understood. Thalassemia patients with similar clinical features and a sound immune system can be used as good in vivo models to study immune recovery after transplantation.

In addition to the same disease, the study had a high degree of concordance on conditioning regimens, GVHD prophylaxis and transfused cell doses compared to other studies. It noticed 
that a significant association between PTF levels and CPE in 7/8 HLA-matched HSCT. When the PTF level in the entire study cohort was less than $2594 \mathrm{ng} / \mathrm{ml}$, the probability of CPE was significantly reduced. In the enrolled HSCT study, one HLA alleles mismatch increased the risk of developing CPE, particularly involving multiple loci mismatches $[16,17]$. It is well-known that one of the key factors influencing HSCT results is HLA matched degree at HLA-A, B, C and /or DRB1 loci [18]. The study demonstrated that high levels of PTF worsened the outcome of thalassemia mismatched HSCT and increased the incidence of CPE.

Although serum ferritin concentration is not the best indicator of total iron overload in the body, it remains the most cost-effective and practical screening method for estimating iron overload [16]. Ferritin concentrations have been accepted as a predictor of iron overload in patients undergoing HSCT $[19,20]$. A recent report noted that greater than $685 \mathrm{ng} / \mathrm{ml}$ of PTF was associated with a significant reduction in overall and recurrence-free survival [21]. Altes et al[13] have shown that ferritin concentrations greater than $3000 \mathrm{ng} / \mathrm{ml}$ and transferrin saturation greater than $100 \%$ upon transplantation are associated with post-HSCT toxin-related death. Majhail et al [22] PTF significantly increased the risk of early non-recurrent death after HSCT. In a prospective study, Pullarkat et al[23] demonstrated that iron overload assessed using ferritin prior to transplantation is an effective predictor of mortality and overall survival at day 100 following HSCT. In addition to being a pre-transplant risk factor leading to early transplant complications and reduced survival, iron overload may also confound clinical findings by mimicking the symptoms of several post-transplant complications or increase the incidence of several post-transplant complications [24].

As a heteropolymer containing $24 \mathrm{H}$ - and L-type subunits, ferritin has been considered as an immunomodulatory [25]. Several evidences indicated that ferritin could inhibit the proliferation of $\mathrm{T}$ cells, block the maturation of $\mathrm{B}$ cells and inhibit the proliferation of bone marrow cells by reacting to mitogens. H-ferritin has been proposed to induce regulatory $\mathrm{T}$ cells to produce interleukin-10 and thereby suppress the immune response [26]. Based on these findings, multivariate analysis of high ferritin demonstrated that the increased incidence of CPE in thalassemia patients may be related to the inhibitory effect of ferritin on the adaptive immune response. Ferritin levels are not only triggered by iron-overload, but also included other factors such as inflammation, infection and liver damage [27]. So, these factors should be considered in using the conclusions of this study. It will be helpful to more accurately analyze the association between PTF and CPE incidence using MRI to measure objectively liver iron content.

\section{Conclusion}

In summary, these results suggested that high PTF levels increased the incidence of CPE after 7/8 HLA-matched HSCT for thalassemia patients. PTF of $2500 \mathrm{ng} / \mathrm{ml}$ is a threshold value that associated with a high or low incidence of CPE. PTF level should be reduced below the threshold value when patients with thalassemia received 7/8 HLA-matched HSCT. In the practice, it found that there were more patients with CPE in thalassemia HSCT. For finding out what factors may cause CPE besides HLA mismatch, here, the study focused on and analyzed retrospectively the effect of pre-transplant ferritin (PTF) on CPE in 7/8 matched thalassemia transplant.

\section{References}

[1] Rachmilewitz, E.A. and P.J. Giardina, How I treat thalassemia. Blood, 2011. 118(13): p. 3479-88.

[2] Elborai, Y., A. Uwumugambi, and L. Lehmann, Hematopoietic stem cell transplantation for thalassemia. Immunotherapy, 2012. 4(9): p. 947-56.

[3] Modell, B., et al., Improved survival of thalassaemia major in the UK and relation to $T 2 *$ cardiovascular magnetic resonance. J Cardiovasc Magn Reson, 2008. 10: p. 42.

[4] Ferrari, G., M. Cavazzana, and F. Mavilio, Gene Therapy Approaches to Hemoglobinopathies. Hematol Oncol Clin North Am, 2017. 31(5): p. 835-852.

[5] Baronciani, D., et al., Hemopoietic stem cell transplantation in thalassemia: a report from the European Society for Blood and Bone Marrow Transplantation Hemoglobinopathy Registry, 2000-2010. Bone Marrow Transplant, 2016. 51(4): p. 536-41.

[6] Gaziev, J., et al., Bone marrow transplantation for thalassemia from alternative related donors: improved outcomes with a new approach. Blood, 2013.

[7] Jaing, T.H., et al., Unrelated cord blood transplantation for thalassaemia: a single-institution experience of 35 patients. Bone Marrow Transplant, 2012. 47(1): p. 33-9.

[8] Bakhshi, S. and B. Biswas, Cytomegalovirus in hematological malignancies. Indian Pediatr, 2013. 50(2): p. 193-4.

[9] Hsu, W.Y., et al., Successful management of multilineage autoimmune cytopenia complicated with severe infection and deep vein thrombosis in a patient with Hodgkin lymphoma post-autologous hematopoietic stem cell transplantation. Pediatr Transplant, 2016. 20(1): p. 168-71.

[10] Waespe, N., U. Zeilhofer, and T. Gungor, Treatment-refractory multi-lineage autoimmune cytopenia after unrelated cord blood transplantation: remission after combined bortezomib and vincristine treatment. Pediatr Blood Cancer, 2014. 61(11): p. 2112-4.

[11] Teachey, D.T. and M.P. Lambert, Diagnosis and management of autoimmune cytopenias in childhood. Pediatr Clin North Am, 2013. 60(6): p. 1489-511.

[12] Li, C., et al., A novel conditioning regimen improves outcomes in beta-thalassemia major patients using unrelated donor peripheral blood stem cell transplantation. Blood, 2012. 120(19): p. 3875-81.

[13] Altes, A., et al., Iron overload might increase transplant-related mortality in haematopoietic stem cell transplantation. Bone Marrow Transplant, 2002. 29(12): p. 987-9. 
[14] Holbro, A., M. Abinun, and T. Daikeler, Management of autoimmune diseases after haematopoietic stem cell transplantation. Br J Haematol, 2012. 157(3): p. 281-90.

[15] Bhatt, V., et al., Autoimmune hemolysis and immune thrombocytopenic purpura after cord blood transplantation may be life-threatening and warrants early therapy with rituximab. Bone Marrow Transplant, 2016. 51(12): p. $1579-1583$

[16] Thambiah, S.C., et al., Pre-transplantation serum ferritin as a prognostic marker in allogeneic haemopoietic stem cell transplant patients in a tertiary care hospital in Malaysia. Natl Med J India, 2016. 29(0970-258X (Print)): p. 136-140.

[17] Shaw, B.E., et al., The impact of HLA genotyping on survival following unrelated donor haematopoietic stem cell transplantation. $\mathrm{Br} \mathrm{J}$ Haemato, 2010. 150(1365-2141 (Electronic)): p. 251-8.

[18] Oran, B., et al., Better allele-level matching improves transplant-related mortality after double cord blood transplantation. Haematologica, 2015. 100(10)(1592-8721 (Electronic)): p. 1361-70.

[19] de Witte, T., The role of iron in patients after bone marrow transplantation. Blood Rev, 2008. 22(1532-1681 (Electronic)): p. 22-8.

[20] Sucak, G.T., et al., Iron overload: predictor of adverse outcome in hematopoietic stem cell transplantation. Transplant Proc, 2010. 42 (5) (1873-2623 (Electronic)): p. 1841-8.
[21] Kataoka, K., et al., Influence of pretransplantation serum ferritin on nonrelapse mortality after myeloablative and nonmyeloablative allogeneic hematopoietic stem cell transplantation. Biol Blood Marrow Transplant, 2009. 15(2)(1523-6536 (Electronic)): p. 195-204.

[22] Majhail, N.S., L.J. Lazarus Hm Fau - Burns, and L.J. Burns, Iron overload in hematopoietic cell transplantation. Bone Marrow Transplant, 2008 41(0268-3369 (Print)): p. 997-1003.

[23] Pullarkat, V., et al., Iron overload adversely affects outcome of allogeneic hematopoietic cell transplantation. Bone Marrow Transplant, 2008. 42(12)(1476-5365 (Electronic)): p. 799-805.

[24] Mahindra, A., et al., Elevated ferritin is associated with relapse after autologous hematopoietic stem cell transplantation for lymphoma. Biol Blood Marrow Transplant, 2008 14(1523-6536 (Electronic)): p. 1239-44.

[25] Recalcati, S., et al., New functions for an iron storage protein: the role of ferritin in immunity and autoimmunity. $\mathrm{J}$ Autoimmun, 2008. 30(0896-8411 (Print)): p. 84-9.

[26] Gray, C.P., P. Arosio P Fau - Hersey, and P. Hersey, Heavy chain ferritin activates regulatory $T$ cells by induction of changes in dendritic cells. Blood, 2002. 99(9)(0006-4971 (Print)): p. 3326-34.

[27] Sakamoto, S., et al., Differing impacts of pretransplant serum ferritin and $C$-reactive protein levels on the incidence of chronic graft-versus-host disease after allogeneic hematopoietic stem cell transplantation. Int J Hematol, 2013. 97(1865-3774 (Electronic)): p. 109-16. 